\title{
ORIGEN Y TRANSPORTE DE SEDIMENTOS EN ESTUARIOS
}

Por:

\author{
Homero Cabrera Muro*
}

Ciencias Marinas Vol. 2 Núm. I

- Domicilio Actual: School of Oceanography, Oregon Stata University

Corvalis, Or. 97331. U. S. A.

\section{RESUMEN}

Recientemente, ha surgido un fuerte in terés por el desarrollo de las zonas costeras. Entre los medios ambientes costeros, los estuarios son particularmente importantes para la utilización industrial, as como para maricultivo, actividades recreacionales, navegación y desarrollo portuario Un estuario es definido por Pritchard (1955) sobre tres características básicas: II un cuerpo de agua semicerrado, 2) libre circulación con el océano abierto, y 3) ocurre la mezcla de dos tipos de agua, agua de mar y agua dulce. De acuerdo con eşta definición se excluyen características morfológicas de la costa, además los estuarios son clasificados por Pritchard (1955) de acuerdo a modelos de circulación y distribueión de salinidad en: mezclados, parcialmente mezclados y estuarios estratificados.

También, el origen de los sedimentos en los estuarios ha sido ampliamente estudiado durante los últimos 25 años. Mea de (1972) considera tros fuentes de sedimentos: de origen fluvial, áreas litorales, y márgenes del estuario mismo. En la mayoría de los estuarios estudiados en detal le, la descarga de los ríos se ha mostrado como la fuente principal de sedimento. Los procesos de transporte y posterior depositación en un estuario son fuertemente afectados por los modelos de circulación y distribución de la salinidad así como por el estado de agregación del material sus. pendido. Un factor adicional en la distribución de los sedimentos, incluye los organismos filtradores, así como la coalición y cohesión los cuales tiender. a agregar las partículas en cierto grado.

En un estuario se reconocen tres ambientes de depósito, de acuerdo con las caracteristicas hidrodinámicas y las condiciones de transporte y depositación prevalecientes. Estos ambientes de depósito san llamados fluvial, marino y`transicional.

Una dificultad en el estudio de los sistemas estuarinos es que la mayoría de los estuarios estudiados intensivamente, han sido alterados en cierto grado por las actividades humanas. Esto debe ser considerado en el estudio de los medios ambientes estuarinos específicos. Solamente a través de la comparación con áreas estuarinas similares y no alteradas. se pueden separar claramente los efectos naturales y artificiales. A menudo esta distinción no ha sido hecha. Recientemente, sin embargo, en la Universidad del Estado de Oregon, se realizan estudios para identificar los mecanismos que perturban los patrones de transporte y depositación de sedimentos er los estuarios.

\section{ABSTRACT}

Among the coastal environments, estuaries are particularly important for industrial utilization, for mariculture, navigation, port development as well as for many recreational activities. For this reason it is critical that a thorough understanding of the dynamics of estuarine systems be known.

An estuary is defined by Pritchard (1955) based on three basic features: 11 a semienclosed coastal body of water, 2) free connection with the open sea, and 3) the mixing of two kinds of water, sea water and fresh water. According to this definition, other coastal features are excluded. In addition, the estuaries are further classified by Pritchard (1955) according to their circulation patterns and salinitv distribu. tion into well-mixed, partially mixed, and stratified estuaries. 
Also, the origin of the sediments in estuaries has been widely studied during the last 25 years. Meade $\{1972\}$ considers three sources of sediment: 1$\}$ river borne sediments, 2 off-shore and littoral areas, and 3) the shoreline of the estuary itself. In most of the estuaries studied in detail, the upland discharge has been shown to be the principal source of sediment. Further, transport and deposition of suspended material within estuaries is strongly affected by the circulation patterns and salinity distribution as well as the state of aggregation of the suspended matter. Additional factor on sediment distribution include filter-feeding organisms and collision and cohesion processes which tend to aggregate particles to some extent.

Three different realrus of deposition are recognized within an estuary, uccording to the hydrodynamic conditions and the transport and deposition of the sediments observed. These realms are called fluviatile, marine, and transitional.

A difficulty in studying estuary systems is that most of the estuaries intensively studied have atready been altered to some extent by man'n activities. This must be considered when dealing with specific estuarine areas can the natural and artificial results be seporated clearly. Otten, this distinction is not made. Recently, however, attempts to identify the mechanisms that disturb sediment patterns in estuaries, are being made at Oregon State University.

\section{INTRODUCCION}

El desarrollo de las áreas costeras ha sido muy rápido en años recientes. Los varios factores que definen un estuario hacen de él un sitio deseable para industrialización ya que proveen una fuente de agua dulce, y un medio para el flujo de desechos. Los estuarios también son un medio ambiente conveniente para el cultivo de algunns especies de moluscos, crustáceos y peces, y su uso como área recreacional se ha incrementado marcadamente.

Los estuarios no son un medio ambiente oceánico ni de agua dulce, y presentan numerosos problemas de carácter único. En ellos la variabilidad, es mucho mayor que en el océano abierto. La circulación, y las propiedades físicas y químicas del agua cambian rápidamente debido a variaciones en el rango de mareas, descarga fluvial, y condiciones meteorológicas. La variación estacional del rango de marea y la descarga fluvial en un estuario también influencían fuertemente la distribución espacial y temporal de los parómetros físico-químicos así como también el material suspendido. La circulación, que mueve agua salina del océano hacia dentro del estuario y mezcla esta agua con el agua de origen fluvial que se mueve hacia el océano, es importante en la determinación y patrón de movimiento de todo el material sus- pendido y disuelto presente en un estuario. Las concentraciones de sales, contaminantes, oxígeno, plancton, nutrientes y sedimentos son en parte controlados por este patrón de movimiento y pueden variar grandemente en cortos períodos.

El propósito d este trabajo es revisar las relaciones observadas entre la circulación y el movimiento de los sedimentos.

\section{ESTUARIO: UNA DEFINICION}

Los estuarios han sido definidos desde diferentes puntos de vista (ej. geológico, biológicol, pero la mayoría de las definiciones son muy exclusivas. La definición más completa de un estuario es la de Pritchard (1955) quien define a un estuario como "un cuerpo costero de agua semi-cerrado con libre circulación de agua con el océano, en el cual ocurre agua salina mezclada con agua de origen fluvial". De acuerdo con esta definición, las grandes áreas costeras tales como el Báltico, el Golfo de Bothnia, y el Golfo de Finlandia son excluidas. La segunda restricción es el libre intercambio con el océano, implicando que la boca del estuario debe permitir un libre intercambio entre el estuario y el océano. Esta restricción elimina aquellos cuerpos semi-cerrados de agua costera donde la hoca no es suficientemente grande 
para permitir esta circulación. Este tipo de morfología costera puede ser llamada estuario de barra, estuario ciego o laguna estuarina (Bird, 1968) pero Pritchard (1955) prefiere llamarlos lagunas costeras para evitar una terminología confusa. La tercera característica de un estuario es la mezcla de agua oceánica y agua dulce. La dilución del agua de mar por el agua dulce produce gradientes de densidad que originan la característica circulación estaurina.

\section{CLASIFICACION ESTUARINA}

Los estuarios han sido clasificados en un sistema generalmente aceptado, desarrollado por Pritchard (1955) de acuerdo a su circulación y distribución de salinidad.. En este sistema un estuario dado puede cambiar de un tipo a otro durante un aro de acuerdo con los cambios observados en la distribución de salinidad y razón de fiujo en cada estación. La razón de flujo es definida como la razón de la descarga fluvial durante un medio ciclo de marea de 12.4 horas al prisma de marea, el cual es en volumen entre la media marea alta, y la media marea baja (Pritchard. 1955).

$\underset{\text { flujo }}{\text { Razón }}=\frac{\begin{array}{c}\text { Descarga fluvial } \\ \text { durante medio ciclo de marea }\end{array}}{\text { Prisma de marea }}$

Altas descargas fluviales, con un valor de razón de flujo del orden de uno - más, proveen un gran volumen de agua duice en la superficie que ayuda a mantener fuertes gradientes de salinidad en estuarios estratificados Tipo A (Fig. 1). Con pequeña descarga fluvial y una razón de flujo de 0.2 a 0.5 , un estuario es parcialmente mezclado Tipo B (Fig. 2). Cuando la descarga del río es baja y la razón de flujo es menor que 0.1 , el estuario está probablemente bien mezclado y verticalmente homogéneo Tipo C (Fig. 3). Como un ejemplo, la razón de flujo en el estuario Columbia va de valores mayores que uno durante el máximo de precipitación y escurrimiento en invierno a valores de $0.1-1.0$ en verano, cambiando su circulación de es- tratifcada a parcialmente mezclada. A continuación se dan algunas de las características más determinantes para producir diferentes tipos de estuarios:
A. Estratificado
1 Bajo rango de marea
2. Alta descarga fluvial
3. Canal profundo
4. Diferencia en salinidad $20 \%$
5. Razón de flujo mayor que $I$

B Parcialmente mezciado
1. Rango de mareas moderado
2. Descarga fluvial moderado
3. Profundidad moderado
4. Diferencia de salinidad $4, c-19 \%$
5. Razón de flujo 0.1 - 1.0

C. Verticalmente homogéneo

1. Alto rango de marea

2. Baja descarga fluvial

3 Pequeñas profundidades

4. Diferencia de salinidad $3 \%$

ias salinidades superficiales y de fondo son observadas en una sección transversal del estuario donde la salinidad promedio es $17 \%$ (Burt y McAlister, 1959). Estos autores explican los tres tipos de estuarios $(A, B, C)$ con definiciones un poco diferentes. Una circulación estratificada consiste de una capa de agua de origen fluvial sobre otra capa de origen marino. El agua dulce se distribuye y circula en la superficie hacia el océano mientras que el agua marina, más densa se mueve por el fondo hacia dentro del estuario, formando un frente de salinidad, que se extiende corriente arriba alguna distancia que depende de la razón de flujo. Entre la interfase de agua salina y fluvial el agua de mar se mezcla verticalmente con el agua dulce $y$ es movida corriente abajo, hacia el océano en forma diluida. Debido a esta pérdida de agua salina debe existir una circulación corriente arriba en la capa de agua salina, para establecer un balance (Fig. 1).

El segundo tipo de estuario se desarrolla cuando la circulación se torna moderada. Debido a la mezcla vertical en este tipo de estuario, el gradiente de salinidad es débil, sin embargo, en una localidad dada, el agua cerca del 


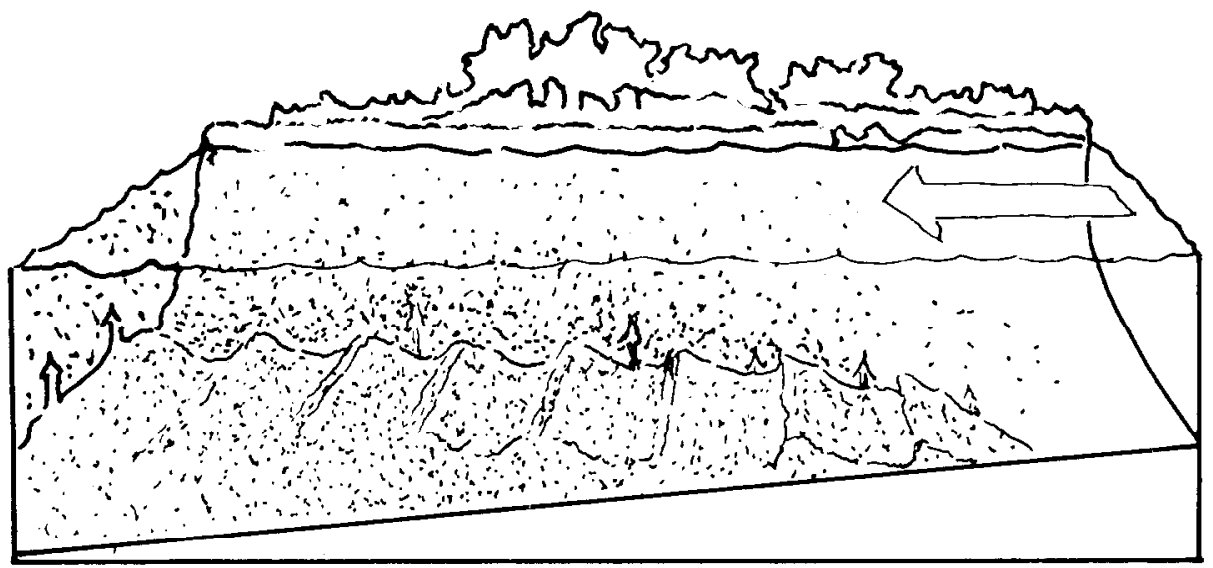

Figura 1. Estuario Tipor

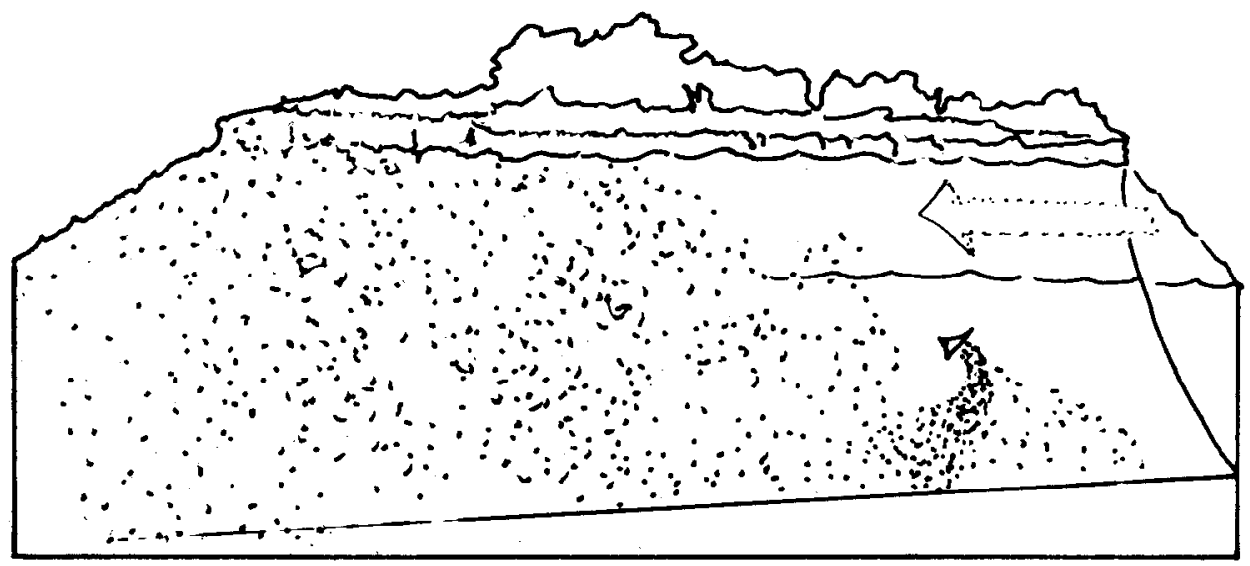

Figura 2. Estuario Tipo B

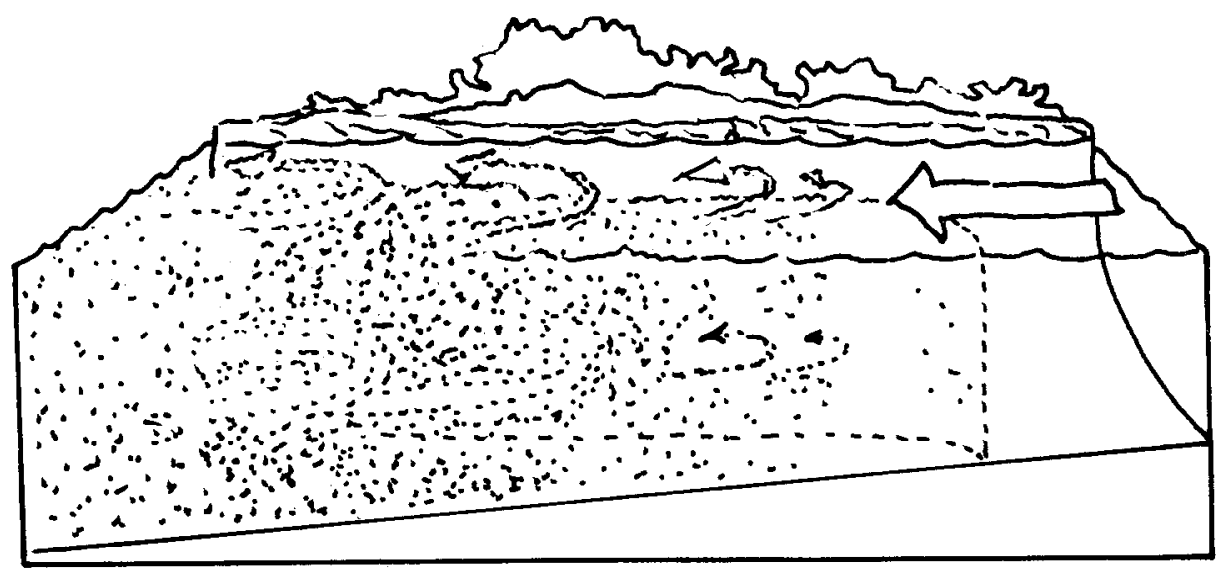

Figura 3. Estuario Tipo C 
fondo es más salina que aquella de la superficie, las corrientes más fuertes son encontradas cerca del fondo y dirigidas hacia adentro del estuario. Un ejemplo de estuario parcialmente mezclado es el estuario Columbia. Es interesante notar que un contaminante que entra a un estuario de este tipo, cerca del fondo puede viajar un número de millas corriente arriba, antes de ser mezclado completamente con la capa superficial (Fig. 2).

Un sistema verticalmente homogéneo, se desarrolla, bajo la influencia de altas mareas, escurrimientos escasos, y poca profundidad. Las restricciones $u$ obstrucciones en la topografía del canal también tienden a desarrollar este tipo de circulación. Varios estuarios de Oregon, E.U.A., se tornan parciaimente homogéneos al finalizar el verano y comenzar el otoño, período normal de mínima precipitación. Con este tipo de flujo y distribución de salinidad existe un movimiento neto de agua hacia el océano, con el movimiento de mareas superpuesto. Ocasionalmente, la marea entrante puede fluir más fuertemente en la superficie $y$ moverse por encima de la capa de agua un poco menos densa (debido a la mezcla) y menos salina en el fondo. Esto resulta en una condición inestable, la cual incrementa la turbulencia y mezcla que hace este tipo de estuarios verticalmente homogéneos. Burt y Queen (1957) dan una discusión más completa sobre esta forma de mezcla en estuarios.

Los diferentes tipos de circulación afectan la naturaleza y razón de sedimentación, así como la mezcla por mareas provee un medio de transporte para material suspendido o disuelto en el astuario.

\section{ORIGEN DE LOS SEDIMENTOS EN ESTUARIOS}

Meade (1972) considera como fuentes primarias de sedimentos estuarinos las siguientes: fluvial, áreas litorales, y márgenes estuarinas. Sin embargo, en la mayoría de los estuarios que han sido estudiados intensivamente, la descarga fluvial se ha mostrado como la fuente principal de sedimentos, principalmente en la porción más interna del estuario, mien- tras que en las partes medias y cerca de la boca, la erosión costera es la fuente mayor de sedimentos acarreados hacia dentro del estuario. El grado en que cada una de estas fuentes sedimentarias prevalecerá depende de los procesos dinámicos de circulación y mezcla.

\section{SEDIMENTOS DE ORIGEN MARINO}

Las bocas de numerosos estuarios están particularmente azolvadas con sedimentos derivados de islas de barrera 0 de la plataforma continental adyacente, la mayoría de estos sedimentos son arena. En áreas donde existe esta fuente de arena, el movimiento corriente arriba de la capa inferior que caracteriza los estuarios tipo $A$ y $B$ puede ser un mecanismo importante para el transporte de estos sedimentos hacia dentro del estuario. Como un ejemplo, Crommelin (1940) y Van Stratten (1960) estudiaron el tamaño de los sedimentos en el estuario Ems en Alemania y concluyeron que la mayoría de los sedimentos provinieron del Mar del Norte. Otro estuario donde el mar ha sido sugerido como la fuente principal de sedimento es el Mersey. Esto fue probado por Steers (1967) quien usó trazadores radioactivos para demostrar el movimiento hacia dentro del estuario de sedimentos provinientes de bancos de arena localizados frente a la boca del estuario. En Oregon, las evidencias mi. neralógicas indican sedimentos de origen marino en sus estuarios (Kulm y Byrne, 1967).

\section{LOS RIOS COMO FUENTE DE SEDIMENTOS}

Como fue señalado antes, en la mayoría de los estuarios la mayor parte de los sedimentos han sido derivados de fuentes fluviales. La cantidad de sedimentos descargada por un río depende de factores tales como la variación temporal del flujo y el tipo de suelos en la cuenca de drenaje. Los ríos mueven sedimentos con amplio rango en tamaño, desde arcillas a grava, en suspensión y tracción. En algunos ríos, tal como el río Potomac, las fuertes descargas de sedimentos ocurren durante pocos días, cuando los escurrimientos de primavera son más fuertes. El grueso de esta descarga 
es atrapada en el estuario, y las proporciones de los diferentes tamaños de sedimento presentes, varían estacionalmente $y$ de un estuario a otro (Schubel, 1964). En base anual Haushild (1966) estimó que los sedimentos transportados al estuario Columbia, durante 1963 consistió en 2.4 millones de toneladas de arena, $3.9 \mathrm{mi}-$ llones de tons. de arcilla y 1.5 billones de tons. de limo.

Otros factores que afectan la cantidad de sedimentos descargados por un río, son las actividades humanas. La limpieza de la tierra para el cultivo y la construcción constituyen una posible fuente de sedimentos mientras que la conservación de los suelos decrece esta fuente.

\section{EROSION COSTERA DE LAS MARGENES ESTAURINAS COMO FUENTES DE SEDIMENTOS}

En la parte más interna del estuario Chesapeake, Schubel (1971) encontrố que los sedimentos derivados por autodigestión de las márgenes estaufinás fue entre 50-100\% de la contribución sedinenentaria fluvial. Por tanto, en algunos casos, este proceso puede ser una fuente mayor de sedimentos, a menudo no evaluada.

\section{MATERIALES EN SUSPENSION}

Parte de los sedimentos que han sido contribuidos por los ríos y otras fuentes primarias forman la carga en suspensión disponible para su transporte y depositación en el ambiente estaurino. Esencialmente todas las partículas suspendidas en aguas estaurinas a bajas velocidades son más finas que 100 micrones (Meade, 1972).

En estuarios fuartemente estratificadoss, la earacferística más importante de la distribución da la materia suspendida, es la zona horizontal de máxima concentración logellizada en la parte más interna de la intrusión sulitial. Esta zona de máxima concentración aparentemente de= pende del flujo bidireccional para su existencia, y laconcentración de material suspendido refleja los cambios en posición del frente salino durante los estados de descarga fluvial. El frente salino puede ser movido hacia la boca del estuario y las zonas de máxima concentración de material suspendido se presenta en el fondo del canal, pero cuando la intrusión de sal penetra al estuario debido a altas mareas, la máxima concentración queda situada nuevamente por encima de la intrusión salina (Meade, 1972).

En adición, las partículas suspendidas que se mueven hacia el mar cerca de la interfase de agua salina y dulce, pueden ser atrapadas por turbulencia y eventualmente moverse corriente arriba. Estas partículas pueden cruzar la interfase nuevamente y alcanzar la capa superior, repitiendo el ciclo completo varias veces. La dirección y magnitud del movimianto resultante de las partículas depeñdera de la velocidad relativa del flujo de la capa superior con respecto a la capa inferior. Entonces, la turbulencia en la interface puede ayudar a mantener los sedimentos en suspensión y por medio de este mecanismo pueden ser acarreados sabre la capa de agua salina, hasta alcanzar las aguas costeras.

En estuarios moderadamente estratificados un máximo de concentración de materia suspendida también persistente cerca del frente salino (Meade, 1972). Esta característica ha sido llamada máximo de turbidez y ha sido observada en numerosos estuarios. El máximo def furbidez ha sido atribuido a la floculación de los edimentos fluviales dispersos, cuando se encuentran en su transporte con intrusión salina (Postma y Kalle, 1955; Freitag, 1960).

La floculación depende para su ocurrencia en la coalición y cohesión. Altas concentraciones y mezcla intensiva provocan un incremento del número de coaliciones entre las partículas y fortalece la cohesión entre ellas, pero este proceso no puede producir independientemente las altas concentraciones de sedimentos suspendidos observadas en la zona de máxima turbidez. Schubel (1971) considera que la floculación es impórtante en esta zona como un resultado de la máxima concentración, pero no como una causa de ella.

La agregación de material suspendido rambién puede ser debida a los orgarismos. Los organismos filtradores que 
viven en estuarios aglomeran partículas finas suspendidas, en ruerpos cuya velocidad de depositación es varias veces más grande, que la de las partículas constituyentes. Si se considera el número de organismos filtradores presentes en un estuario, probablemente pueda decirse que tódas las partículas finas de los sedimentos de fondo han sido removidas - de alguna manera afectadas por más de un organismo. Sin embargo, la importancia relativa de este proceso con respecto a otros no es bien conocida (Meade, 1971).

En estuarios verticalmente homogéneos, los sedimentos se mueven de manera similar que en los estuarios moderadanente estratificacios, aungue el proceso puede ser más sutil. Por ejemplo, en el estucrio de Thames, en el cual la salinidad escasamente difiere en una parte pór mil de la superficie al fondo en cualquir estación del año (lnglis y Allen, 1957), esta débil diferencia vertical en densidad es aparentemente suficiente para causar el flujo predominante hacia adentro del estuario y cerca del fondo. Este fluio acarrea sedimentos y es más fuerte durante las mareas vivas y más débil o inclusive dirigido hacia el mar durante las mareas muertas.

En adición en el estuario del Thames el efecto de Cariolis es también expresado más fuertemente. En el estuario Thames, esto causa que ios sedimentos se muevan predominantemente hacia el mar, en el lado norte del estuario, mientras que en el lado sur lo es hacia adentro del estuario (Inglis y Allen, 1957), el movimiento hacia tierra de los sedimentos ha sido demostrado en otros estuarios (Bahía de Liverpool) por medio de trazadores radioactivos.

\section{AMBIENTES DE DEPOSITO}

Las condiciones hidrodinámicas, fuentes sedimentarias y los procesos de transporte y depositación de sedimentos en un estuario, crean diferentes ambientes de depósito. Kulm y Byrne (1966) observaron tres ambientes de depósito mayores, en el estuario del Yaquina. Estos ambientes han sido llamados marino, fluvial, y un ambiente transicional, marino-fluvial.
El marino, que incluye las playas y dunas adyacentes a la bahía, es"tipificado por agua de origen marino $y$ por la acción vigorosa de las olas y mareas. Los sedimentos estuarinos de este ambiente son similares a los sedimentos de dunas y playa adyacentes, consisten de arena media a fina, subangular a subredondeada, y bien sorteada. Estas arenas se distinguen además, por la asociación de minerales pesados, los cuales incluyen abundantes piroxenas, y minerales metamórficos tales como kyanita, sillimanita, y staurolita.

El medio fluvial ocurre corriente arriba donde las condiciones de agua dulce prevalecen. Los sedimentos pobremente sorteados, angulares a subangulares presentan un rango en tamaño de arcilla a arena coarzosa. Presentan también una asociación de minerales pesados e incluyen biotita, muscovita, hematita y limonita.

El medio ambiente transicional está entre el fluvial y el marino así como un amplio rango en la textura y mineralogía presente. Los sedimentos están bien - pobremente sorteados y sus partículas van de angulares a subredondeadas, con variedad de tamaño de arcilla a arena media. La amplia variación mineralógica resulta de la mezcla de ședimentos provinientes de los medios marino y fluvial, así como de la posición cambiante de la intrusión salina.

\section{DRAGADO Y OTROS IMPACTOS DE ACTIVIDADES HUMANAS}

La razón de perturbación de los sedimentos en un estuario y su relación con actividades humanas, especialmente el dragado, está siendo estudiado a la fecha por un grupo de investigación en áreas tales cono: caracterización sistemática de propiedades estuarinas individuales; interacción con grupos de usuaios y desarrollo de conceptos y técnicas para determinar los efectos del dragado y otras alteraciones en los estuarios.

Esta investigación pretende identificar cada uno de los mecanismos que tienden a alterar los patrones de sedimentos estuarinos. Esos factores son identificados y caracterizados con respecto a la frecuencia de ocurrencia y las alteraciones 
producidas en los sedimentos de este medio ambiente.

\section{CONCLUSIONES}

De acuerdo a los patrones y distribución de salinidad los estuarios son clasificados como verticalmente homogéneos, parcialmente mezclados y estratificados. El material transportado en suspensión o tracción tiene como fuente primaria los sedimentos de ríos, zona litoral y en cierto grado las márgenes del estuario. Sin embargo, en la mayoría de los estuarios la fuente principal de sedimentos es la descarga fluvial. Los sedimentos marinos se han observado principalmente en la boca y partes bajas de un estuario

Se observan tres ambientes de depó. sito, controlados por las condiciones hidrodinámicas, el transporte y depositación de los sedimentos. El ambiente fluvial está caracterizado por agua dulce $y$ material anguloso pobremente sorteado. El ambiente transicional está situado corriente abajo del fluvial $y$ es caracterizado por mezcla de agua salina $y$ dulce con sedimentos bien o pobremente sorteados. Presenta amplias variaciones en la mineralogía como resultado de la mezcla de los ambientes marino-fluvial. El ambiente marino está cerca de la boca $y$ en él se observa agua salina y los sedimentos están bien sorteados, compuestos de arena fina.

La mayoría de los estuarios han sido estudiados, también han sido dragados - de algún modo alterados por actividades humanas (Meade, 1971). Consecuentemente, se necesitan más estudios en otros estuarios donde la influencia del hombre no es fuerte, para separar las condiciones artificiales de las naturales en este medio ambiente.

\section{AGRADECIMIENTOS}

Expreso mi agradecimiento al Dr. Paul Komar y $\mathrm{N}$. Dahm por sus útiles críticas y sugerencias. Los comentarios del Dr. Slotta fueron también fructíferos. Doy gracias al Consejo Nacional de Ciencia y Tecnología de la República Mexicana por su asistencia becaria.
BIBLIOGRAFIA

Bird, E. C. F., 1968. Coosts, M.I.T, Press, Cambriage, Bowden, K. F., 1967. Circulation and Diffusion. Estuaries, p. 15-35. American Association for the Advancement of Science. Publicación Núm.' 83. Washington, D. C.

Burt, Wayne V. y John Queen. 1957. Tidal overmixing in estuaries. Science, 126 (3280): 973-974.

Burt, W. V. y W. B. MCAlister 1959. Recent studies in tis? hydrography of Oregon estuaries. Res. Briefs, 7: 14-27.

Crommelin, R. O., 1940. De Herkomst van het zand de Waddenzee. Tiid. Koninkl, Ned. Aardi, Fen., v57: 361-397.

Freitag, D. R., 1960. Soil as a factor in shoaling processes, a literature review, Tech. Bull. 4, Comm. en Tidal Hydraulics, U. S. Army Corps of Engineers, p. $1-47$.

Haushild, W. L., Perkins, R. W., Stevens, H. H., Jr. Dempster, G. R. Jr., y Glenn, J. 1., 1966. Progress report: Radionuclide transport in the Pasco to Vancouver, Washington reach of the Columbia River, July 1962 to September 1963: U. S. Geol. Survey open tile report, 188 p. 1 pl.

Inglis, C. C. Y Allen F. H. 1957. The regimen of the Thames Estuary as affecisd by currents, salinities, and river flow. Inst. Civil Engineers Proc. v. 7. p. 827-878; Disc., v. 8, p. 437-439.

Kulm, L. D. y John V. Byrne. 1967. Sedimentary response to hydrography in an Oregon estuary. OSU Department of Oceanography. Collected Reprints, V. 5: 75-105.

Meade, R., 1972. Transport and deposition of sediments in esfuaries. The Geological Society of America, Inc. Memoir 133.

Neal, T., Victor, 1965. A calculation of flushing time and pollution distribution for the Columbia River estuary. Ph. tests Oregon State University, Dept. of Oceanography.

Postma and K. Kalle, 1955. Die Entstehung von Trueburigszonen in Unterlauf der Fluesse, Deutsche Hyarographische Zeitschieft, v. 8, p. 137-144.

Pritchard, D. W. 1955. Estuarine circulation patterns. Proc. Am. Soc. Civil Engrs. 81: 1-11.

Schubel, J. R., 1967. On suspended sediment sampling by filtration. Southeastein Geoolgy, V. 8, p. 85-87.

Schubel, J. R. 1971. Suspended sediment discharge of the Susquehanna River at Conowingo Maryland during 1969. Ches. Science.

Schubel, J. R., 1971. Sedimentation in the upper reaches of the Chesapeake Bay. The estuarine environment. AGI short course lecture notes, $p$. VII (1-3)). Apnerican Gzoloaical Institute. Washingron, D. C.

Sluria, L. S., 1974. An examination of some physical and biological impacts of dredging in estuaries. Interim Progress Report, p. 60-81. Interdisciplinary studies of the School of Engineering and Oceanography. OsU.

Smith, D. B. y Parsons, T. V., 1967. Radioisotope techniques for determining silt movement from spoil grounds in the firth of forth: Internat. Atomic Energy Agency and Internat. Union Geodesy and Geophysics Symposium on lsotopes in Hidrology, Vienna 1966, Proc p. 167-180.

Steers, J. A. 1967. Geomorphology and coastal processes. Estuaries, p. 100-107.. American Association for the Advancement of Science. Publication No. 83. Washington, $D, C$.

Van Startten, -L. M. 1960. Transport and composition of sediments. En: Symposium Ems-Esturium, Nordsee. Verhandel, Konikl. Ned. Geol. Mipnobouwk. Benoot. Geol. Serv. v. 19: 279-292. 